SHEP 93/94-19

hep-ph/9406303

\title{
Unification Constraints in the Next-to-Minimal Supersymmetric Standard Model
}

\author{
T. Elliott, S.F. King * \\ Physics Department, University of Southampton, \\ Southampton SO9 5NH, UK. \\ P.L. White ${ }^{\dagger}$ \\ Theoretical Physics, University of Oxford, \\ 1 Keble Road, Oxford OX1 3NP, UK.
}

(October 17, 2018)

\begin{abstract}
We perform a renormalisation group analysis of the next-to-minimal supersymmetric standard model (NMSSM) based on the following constraints: two-loop gauge coupling unification at a variable scale $M_{X}$, running the gauge couplings through the low energy thresholds; universal soft supersymmetry breaking parameters; correct electroweak symmetry breaking. The phenomenological implications of our results include a standard model-like
\end{abstract}

*Email king@soton.ac.uk

${ }^{\dagger}$ Email plw@thphys.ox.ac.uk 
Higgs boson of mass in the range $70-140 \mathrm{GeV}$.

Typeset using REVTEX 
Supersymmetry (SUSY) [1] is an attractive candidate for new physics beyond the standard model at the TeV scale. The existence of SUSY would greatly ease the naturalness problems associated with the construction of a grand unified theory (GUT) of the strong and electroweak interactions. The basic idea of GUTs is that the gauge couplings, which govern the strength of the strong and electroweak interactions at low energy, are actually equal to some unified coupling $g_{X}$ at some very high scale $M_{X}$ due to their renormalisation group (RG) running [2]. The original motivation for SUSY broken at a TeV was to help to stabilise the Higgs mass against GUT scale quadratic radiative corrections. The combination of SUSY and GUTs has recently found some indirect experimental support due to the accurate measurement of the strong and electroweak couplings on the Z pole by LEP. These measurements are inconsistent with gauge coupling unification if a standard model desert is assumed, but are consistent with unification if a SUSY desert above the TeV scale is assumed [3].

In the first post-LEP analyses [3] the whole SUSY spectrum was either assumed to be degenerate at the scale $M_{S U S Y}$, or smeared around this scale. In reality the SUSY partners may have a complicated spectrum, parametrised by a large number of soft SUSYbreaking parameters, which may spread over one or two orders of magnitude of masses. In order to reduce the number of independent soft SUSY-breaking parameters one may appeal to supergravity or superstring scenarios which involve the notion of SUSY breaking in a hidden sector coupled only gravitationally to our observable sector. By this means, in the minimal supersymmetric standard model (MSSM), one ends up with just four independent soft SUSY breaking parameters: $m_{0}, M_{1 / 2}, A_{0}$ and $B_{0}$ corresponding to the universal soft scalar, gaugino, trilinear and bilinear couplings, respectively. Using these four parameters, together with the top quark Yukawa coupling which plays an important role in driving electroweak symmetry breaking [4], several groups have performed an RG analysis whose goal is to predict the SUSY and Higgs spectrum and then use this spectrum as the basis of a more reliable estimate of gauge coupling unification by running the gauge couplings through the various thresholds from the $\mathrm{Z}$ mass $M_{Z}$ up to a unification scale $M_{X}$ 此, 5]. 
Apart from the constraints of gauge coupling unification and correct electroweak symmetry breaking, there are various other phenomenological and cosmological constraints which may be applied and recent studies have concluded that it is possible to satisfy all these constraints simultaneously [6].

Here we shall consider a slightly different low energy SUSY model, but one which is equally consistent with gauge coupling unification, namely the so-called next-to-minimal supersymmetric standard model (NMSSM) [0 9]. The basic idea of the NMSSM is to add just one extra gauge singlet superfield $N$ to the spectrum of the MSSM, and to replace the $\mu$-term in the MSSM superpotential with a purely cubic superpotential,

$$
\mu H_{1} H_{2} \longrightarrow \lambda N H_{1} H_{2}-\frac{k}{3} N^{3} .
$$

The motivation for this "minimal non-minimal" model is that it solves the so-called $\mu$ problem of the MSSM [10] in the most direct way possible by eliminating the $\mu$-term altogether, replacing its effect by the vacuum expectation value (VEV) $\langle N\rangle=x$, which may be naturally related to the usual Higgs VEVs $<H_{i}>=\nu_{i}$. However there are other solutions to the $\mu$-problem [10]. Also, the inclusion of singlets may cause the destabilisation of the hierarchy if there are strong couplings to super-heavy particles such as Higgs colour triplets [11]. Recently similar effects have been shown to result from non-renormalisable operators suppressed by powers of the Planck mass [12]. The dangerous non-renormalisable operators would require that gravity violate the $Z_{3}$ symmetry which is respected by the renormalisable operators of the theory. Our view is that since these effects are model-dependent the NMSSM is as well motivated as the MSSM and should be studied to the same level of approximation. Only by so doing may the two models be phenomenologically compared when (or if) Higgs bosons and SUSY particles are discovered.

In this Letter we present our first results of a GUT-scale RG analysis of the NMSSM, imposing simultaneously the constraints of correct electroweak symmetry breaking and coupling constant unification. In our analysis we shall drop all quark and lepton Yukawa couplings apart from that of the top quark $h_{t}$. We shall assume a soft SUSY breaking 
potential which gives rise to three universal soft parameters at the $M_{X}$, namely $m_{0}, M_{1 / 2}$, $A_{0}$, mentioned above Ð. We shall use the two-loop gauge and Yukawa SUSY RG equations and one-loop soft SUSY RG equations to integrate from the GUT scale $M_{X}$ to a low energy renormalisation scale $Q$. Below $M_{X}$, the three soft mass parameters evolve into 32 separate soft parameters, corresponding to 3 gaugino masses $M_{i}, 11$ trilinear couplings $A_{i}$ (those of the MSSM plus $A_{\lambda}$ and $A_{k}$ ) and 18 scalar masses $m_{i}^{2}$ (including the soft Higgs masses $m_{H_{i}}^{2}$, $\left.m_{N}^{2}\right)$. We assume no inter-generational mixing, and since we drop Yukawa couplings which are small compared to $h_{t}$, the first and second generation soft masses will run identically. The unification constraints are: $g_{i}^{2}\left(M_{X}\right)=g_{X}^{2}, \lambda\left(M_{X}\right)=\lambda_{0}, k\left(M_{X}\right)=k_{0}, h_{t}\left(M_{X}\right)=h_{t 0}$, $M_{i}\left(M_{X}\right)=M_{1 / 2}, m_{i}^{2}\left(M_{X}\right)=m_{0}^{2}, A_{i}\left(M_{X}\right)=A_{0}$.

In practice we shall scale all dimensionful quantities by appropriate powers of $M_{1 / 2}$, and denote the resulting dimensionless quantities by a tilde. At the low energy $\overline{M S}$ scale $Q$ we find the global minimum of the one-loop effective neutral Higgs potential, scaled by $M_{1 / 2}$, including loops of top quarks and stop squarks [13,14]. We only consider the neutral scalar Higgs potential, since there are simple analytical conditions which test for the presence of squark and slepton VEVs [8]. Although these are not guaranteed to prevent all possible charge or colour breaking minima as discussed by Gunion et al in reference [8], they are sufficient to avoid the most dangerous case of slepton VEVs, while a complete analysis of all possible colour breaking minima in the one loop effective potential is not feasible. We implement these conditions after running to an energy scale of order a typical slepton VEV. In addition we ensure that the mass squared of the charged Higgs remains positive in order to avoid breaking electromagnetism. The result of minimisation yields the scaled VEVs $\tilde{\nu}_{i}, \tilde{x}$, from which the condition $\nu=174 \mathrm{GeV}$ fixes $M_{1 / 2}$. Given $M_{1 / 2}$ this enables all the soft parameters and the VEVs to be determined and, as described elsewhere [13,14], it is then straightforward to calculate the top and stop corrections to the Higgs boson mass spectrum.

\footnotetext{
${ }^{1}$ Note that the $B$ parameter does not occur in the NMSSM since we have set $\mu=0$.
} 
The SUSY spectrum is calculated at tree-level using standard results [1], apart from squark masses which we calculate using the one-loop effective potential. There are a number of phenomenological constraints which will cut down the allowed regions of parameter space. We shall require that all sleptons and stops are heavier that $43 \mathrm{GeV}$ and all charginos are heavier than $47 \mathrm{GeV}$. Charged Higgs bosons are required to be heavier than $45 \mathrm{GeV}$. Gluinos and squarks other than stops are required to be heavier than $100 \mathrm{GeV}$. The lightest neutralino is required to be the lightest SUSY particle. The lightest CP-even neutral Higgs boson $h$ is required to satisfy $m_{h} / R_{Z Z h}^{2} \geq 60 \mathrm{GeV}$, where $m_{h}$ is the mass and $R_{Z Z h}$ is the $Z Z h$ coupling scaled by the standard model coupling. We shall require a large top quark mass, $m_{t}>150 \mathrm{GeV}$ [15], which puts a strong restriction on the parameter space of this model. In our analysis we input the gauge couplings $g_{1}\left(M_{Z}\right)$ and $g_{2}\left(M_{Z}\right)$ and run them up through the 24 SUSY and Higgs thresholds to find $M_{X}$ and $g_{X}$, which must of course be consistent with our original input values. By iterating this procedure we obtain solutions which satisfy both the requirements of correct electroweak symmetry breaking and coupling constant unification simultaneously. According to our procedure the parameters $M_{X}$ and $g_{X}$ are determined from $g_{1}\left(M_{Z}\right)$ and $g_{2}\left(M_{Z}\right)$, and so our input parameter set is: $\lambda_{0}, k_{0}, h_{t 0}$, $\tilde{m}_{0}^{2}, \tilde{A}_{0}$, with the value of $M_{1 / 2}$ determined from the VEV. The physically relevant quantities $\alpha_{s}\left(M_{Z}\right)=g_{3}^{2}\left(M_{Z}\right) / 4 \pi, m_{t}, r=x / \nu$ and $\tan \beta=\nu_{2} / \nu_{1}$ are all outputs 2 .

A somewhat similar analysis to that described has been performed in the NMSSM by another group [16 18]. We have properly included the low energy threshold effects in our

\footnotetext{
${ }^{2}$ Our quoted values of $m_{t}$ always refer to the one-loop physical pole mass $m_{t}=m_{t}^{\text {pole }}=m_{t}\left(m_{t}\right)[1+$ $\left.\frac{4}{3 \pi} \alpha_{s}\left(m_{t}\right)\right]$. Also, a word about our conventions is in order. In the convention of Eq.(1), we input positive values of $\lambda_{0}, k_{0}$, and both positive and negative values of $A_{0}$, and look for solutions with both positive and negative VEVs, except that we require $\nu_{2}>0$. Such solutions can always be re-interpreted in terms of purely positive VEVs using the three symmetries of the one-loop effective potential: (i) $\lambda \rightarrow-\lambda, k \rightarrow-k, x \rightarrow-x$, (ii) $\lambda \rightarrow-\lambda, \nu_{1} \rightarrow-\nu_{1}$, (iii) $\nu_{1} \rightarrow-\nu_{1}, \nu_{2} \rightarrow-\nu_{2}$.
} 
unification analysis, whereas in ref. [16 18] such effects were ignored. Since our choice of $\alpha_{3}\left(M_{Z}\right)$ and of $M_{X}$ and $g_{X}$ are determined by the requirements of consistent unification and thus vary with differing spectra, our approach is consequently much more computationally intensive since we have to run down the couplings, minimise the potential, calculate the spectrum, then run the couplings up through the thresholds, iterating this procedure many times in order to obtain a single point in parameter space which is consistent with unification and all the other constraints.

We initially considered a grid of 360,000 values of parameters in the ranges: $\lambda_{0}=0.01-$ 2.0, $k_{0}=0.01-2.0, h_{t 0}=0.5,1,2,3, \tilde{m}_{0}=0.2-5.0, A_{0} / m_{0}=-4$ to +4 , then later investigated the phenomenologically interesting regions in more detail. For the acceptable regions of parameter space we find that $m_{t}>150 \mathrm{GeV}$ is attainable for $k_{0}<\lambda_{0}<h_{t 0}$, with $\lambda_{0}<1$ and $0.5 \lesssim h_{t 0} \lesssim 3$, where the upper limit on $h_{t 0}$ is a requirement of perturbation theory. Although all values of $h_{t 0}$ in this range lead to consistent solutions with $\left|A_{0} / m_{0}\right| \approx 3-4$, for $h_{t 0} \lesssim 0.5$ we also find solutions for smaller $\left|A_{0} / m_{0}\right|$, down to zero. However the cut on the top mass of $m_{t}>150 \mathrm{GeV}$ excludes this region, and so we discuss it no further here. The successful values of $\tilde{m}_{0}$ vary across the whole range, but are correlated with $h_{t 0}$ and $A_{0} / m_{0}$. To be specific, we find for $\left|A_{0} / m_{0}\right| \approx 3$ that $h_{t 0}=0.5,1,3$, is associated with $\tilde{m}_{0} \sim 0.5-1,1-2,2-5$, while smaller values of $\tilde{m}_{0}$ can be obtained for larger $\left|A_{0} / m_{0}\right|$, although the maximum value of $\left|A_{0} / m_{0}\right|$ is about 4 (because of the slepton VEVs constraint). There are also correlations between $\lambda_{0}$ and $k_{0}$ at the unification scale, as discussed later. In the successful regions of parameter space we find $|\tan \beta| \sim 3-20$ (or larger, but our approximations break down here), while $|r|$ is virtually directly proportional to $M_{1 / 2}$, with $|r| \gg 1$ even for small $M_{1 / 2}$. Thus, as in ref. [16], we find that the allowed region of parameter space is characterised by small dimensionless couplings $\left|\lambda_{0}\right|<1,\left|k_{0}\right|<1$ and large singlet $\operatorname{VEV}|x|>1000 \mathrm{GeV}$, corresponding to the approximate MSSM limit of the model [9].

To illustrate the correlation between $\lambda_{0}$ and $k_{0}$, in Fig. 1 we show contours of $h_{t 0}$ in the $\lambda_{0}-k_{0}$ plane with $\tilde{m}_{0}=2, M_{1 / 2}=500 \mathrm{GeV}$ and $\left|A_{0} / m_{0}\right|=3$. Although we could have chosen other values of $M_{1 / 2}$, the qualitative features of Fig.1 would be unaltered, and the 
value of $k_{0}$ would only change by a few per cent, since in this region the value of $M_{1 / 2}$ is a very sensitive function of $\lambda_{0}$ and $k_{0}$. The $h_{t 0}=1,2,3$ contours correspond to $m_{t} \sim 175,185,190$ $\mathrm{GeV}$, respectively, with $|\tan \beta|$ varying from 3-7 and $|r|$ from 100-30, from the lowest to the highest value of $k_{0}$ respectively. Values of $k_{0}$ beyond the ends of the plot are forbidden by the requirements of electroweak symmetry breaking, although the exact ranges of acceptable $k_{0}$ could be altered by varying our choice of $A_{0}$ and $\tilde{m}_{0}$ slightly. Similarly, $m_{t}$ would be altered if we use a different $M_{1 / 2}$ primarily due to the resulting change in $\alpha_{s}\left(M_{Z}\right)$. As observed by other authors [3, [4] we find that larger values of $M_{1 / 2}$ result in smaller values of $\alpha_{s}\left(M_{Z}\right)$. It should be noted that although changing the sign of $A_{0}$ leads to roughly the same output data with a change in the sign of $r$, this is only approximate, and in particular one sign of $A_{0}$ leads to a less restrictive slepton VEV constraint.

We find that, for many choices of our input parameters, there appears to be a certain minimum value of $M_{1 / 2}$ (recall that this is an output parameter). For example, if we impose a cut of $M_{1 / 2} \lesssim 150 \mathrm{GeV}$, then $m_{t}>150 \mathrm{GeV}$ is only possible for $h_{t 0} \approx 1.5-3,\left|A_{0} / m_{0}\right| \approx 3$, $\tilde{m}_{0} \approx 3-5$, corresponding to $m_{t} \sim 185-195 \mathrm{GeV}$. The origin of the minimum value of $M_{1 / 2}$ is due to a different competing global minimum of the one-loop effective potential, which becomes preferred below a minimum $M_{1 / 2}$ value. This happens because the logarithm in the one-loop effective potential depends both on the renormalisation scale $Q$ (which we have so far taken to be $150 \mathrm{GeV}$ ) and on $M_{1 / 2}$. To test the reliability of our results, we have investigated their $Q$-dependence. In doing this we have consistently used the same value of $Q$ both in the RG running of masses and couplings and in the one-loop effective potential, since there is a significant cancellation between the effects of changing $Q$ in these two places.

Although varying $Q$ leads to very little change in the VEVs for any given minimum, it can alter the VEVs substantially by changing which of the minima is deepest (there are often minima with very different VEVs but with similar values of the potential). Given this behaviour, one must be very careful about drawing overly restrictive conclusions based on which of the various minima is preferred for a given region of parameter space. We find that reducing $Q$ will typically lead to a reduction of the minimum value of $M_{1 / 2}$ in 
those regions where small $M_{1 / 2}$ is not possible for $Q=150 \mathrm{GeV}$, but does not otherwise qualitatively change the behaviour. Note that the range of parameters $h_{t 0} \approx 1.5-3$, $\left|A_{0} / m_{0}\right| \approx 3, \tilde{m}_{0} \approx 3-5$ appears to be a "safe" range from the point of view of achieving small values of $M_{1 / 2} \lesssim 150 \mathrm{GeV}$ independently of the choice of $Q$. This dependence on $Q$ is an inherent problem in this model because of the form of the effective potential which has several competing minima. In both our analysis and that of ref. 16 18 electroweak symmetry breaking has been analysed using the one-loop effective potential, including top quarks and stop squarks. In principle the $Q$ dependence could be eliminated by including all particles in the effective potential, and possibly working beyond one-loop. Here we shall restrict ourselves to a discussion of the $Q$-dependence of our results.

We now consider two examples of the sort of Higgs and SUSY spectrum one might typically expect in the constrained NMSSM, the first with relatively large values of $h_{t 0}$ and $\tilde{m}_{0}$, and the second with relatively small values of $h_{t 0}$ and $\tilde{m}_{0}$. These two extreme cases span the complete range of spectra predicted by the constrained NMSSM, subject to the constraint of a large top mass $m_{t}>150 \mathrm{GeV}$.

In Fig. 2 we plot the spectrum as a function of $M_{1 / 2}$ for the case $h_{t 0}=2,\left|A_{0} / m_{0}\right|=3$, $\tilde{m}_{0}=5, \lambda_{0}=0.4$. With $Q=150 \mathrm{GeV}$ we find that $M_{1 / 2}$ may go down to small values, which is not unexpected since these parameters are in the "safe" range $h_{t 0} \approx 1.5-3,\left|A_{0} / m_{0}\right| \approx 3$, $\tilde{m}_{0} \approx 3-5$, referred to above. Note that the experimental constraint that the charginos are heavier than $47 \mathrm{GeV}$ implies that $M_{1 / 2} \gtrsim 70 \mathrm{GeV}$ in this case. For $M_{1 / 2} \approx 100(1000) \mathrm{GeV}$, which is controlled by choosing $k_{0}=0.275(0.300)$, we find $|\tan \beta| \approx 6(8),|r| \approx 13(120)$, $\alpha_{s}\left(M_{Z}\right) \approx 0.121(112), M_{X}=2.1(0.99) \times 10^{16} \mathrm{GeV}, g_{X}=0.71(0.69)$. The lightest CP-even Higgs boson has standard model-like couplings $\left(R_{Z Z h}>0.99\right.$ everywhere $)$ and for $M_{1 / 2} \lesssim 100$ $\mathrm{GeV}$ is in the LEP2 range, as are the lighter chargino and neutralinos which have a much stronger $M_{1 / 2}$ mass dependence. The top quark mass ranges from $m_{t}=193-184 \mathrm{GeV}$, being smaller for larger $M_{1 / 2}$ due to $\alpha_{s}\left(M_{Z}\right)$ being consequently smaller. For $M_{1 / 2} \lesssim 100$ $\mathrm{GeV}$, the lightest stop and gluino are not too much heavier than the top quark, although the remaining sparticles and Higgs bosons are significantly heavier than the top quark. 
In Fig. 3 we plot the spectrum as a function of $M_{1 / 2}$ for the case $h_{t 0}=0.5,\left|A_{0} / m_{0}\right|=$ 4, $\tilde{m}_{0}=0.5, \lambda_{0}=0.1$. These parameters are outside the "safe" range $h_{t 0} \approx 1.5-3$, $\left|A_{0} / m_{0}\right| \approx 3, \tilde{m}_{0} \approx 3-5$, in which small $M_{1 / 2}$ can be achieved independently of the choice of the renormalisation scale $Q$, and so we plot the spectrum for two choices of $Q$. The main effect of changing $Q$ is to change the value of $M_{1 / 2}$ at which the data cuts out. For $Q=150(25) \mathrm{GeV}$, we find the minimum values $M_{1 / 2}=300(125) \mathrm{GeV}$ corresponding to $m_{0}=150(62.5) \mathrm{GeV}$. As in Fig.2, the lightest CP-even Higgs boson has standard model-like couplings, and for a given $M_{1 / 2}$ is even lighter than in Fig.2. For the smaller $M_{1 / 2}$ values which we can obtain with $Q=25 \mathrm{GeV}$ the lighter chargino and neutralinos may be in the LEP2 range. Unlike Fig.2, the left-handed sleptons are now much lighter (due to the smaller value $\tilde{m}_{0}=0.5$ ) while the lighter stop is much heavier (due to the smaller value $h_{t 0}=0.5$ ). The top quark mass has a maximum value of $m_{t}=175 \mathrm{GeV}$ for $M_{1 / 2}=125 \mathrm{GeV}$ and here $|\tan \beta|=3.4$. The gluino in Fig.3 is now the heaviest sparticle, whereas in Fig.2 it was one of the lighter ones. In general the Higgs and sparticle masses in Fig.3 are focussed into a narrower band of masses than in Fig.2, which is a simple result of having $\tilde{m}_{0}=0.5$ rather than $\tilde{m}_{0}=5$.

Finally it is worth comparing our spectra to the spectra discussed in a paper which appeared just as this present article was being finalised [18]. In this most recent analysis the Higgs and SUSY spectrum was presented as as series of scatter plots for masses and couplings. Most of this data and all of ours corresponds to one of the CP-even Higgs bosons being almost pure gauge singlet. Such a decoupled Higgs boson might be expected since the constrained NMSSM is close to the MSSM limit. In the analysis of ref. 18 the decoupled Higgs boson may have a mass either less than or greater than the lighter of the other two CPeven Higgs bosons and when the would-be decoupled Higgs is close in mass to the lighter physical Higgs, strong mixing can occur, leading to two weakly coupled Higgs bosons of the kind discussed in ref. [14]. However, given the range of parameters considered in this paper, we find that the decoupled CP-even Higgs boson is always substantially heavier than the lighter of the two physical CP-even Higgs bosons. Similarly the CP-odd Higgs bosons 
are much heavier than the lightest CP-even Higgs boson. The reason for this difference is simply that in ref. 18] the range of parameters considered exceeds the range considered in this paper. It turns out 5 that in order to bring down the mass of the CP-even singlet sufficiently one requires $\tilde{m}_{0} \ll 0.2, \lambda_{0}<0.01$ and $k_{0} \ll \lambda_{0}$, corresponding to extremely large values of $r \gg 100$. We have checked that, in this region of parameter space, the singlet CP-even Higgs boson does indeed become much lighter, leading to the strong mixing effect mentioned above. However, for the range of parameters considered in the present paper, a standard model-like Higgs boson with a mass in the range $70-140 \mathrm{GeV}$ is preferred.

\section{Acknowledgement}

We are very grateful to Ulrich Ellwanger for his help in correcting an error in an earlier version of this paper.

\footnotetext{
${ }^{3} \mathrm{U}$. Ellwanger, private communication
} 


\section{REFERENCES}

[1] H.P. Nilles, Phys. Rep. 110 (1984) 1; H.E. Haber and G.L. Kane, Phys. Rep. 117 (1985) 75.

[2] H. Georgi, H.R. Quinn and S. Weinberg, Phys. Rev. Lett. 33 (1974) 451.

[3] U. Amaldi, W. de Boer and H. Furstenau, Phys. Lett. 260 B (1991) 447; J. Ellis, S. Kelley and D. V. Nanopoulos, Phys. Lett. 260 B (1991) 131; P. Langacker and M. Luo, Phys. Rev. D44 (1991) 817.

[4] G.G. Ross and R.G. Roberts, Nucl. Phys. B 377 (1992) 571.

[5] R. Arnowitt and P. Nath, Phys. Lett. B 287 (1992) 89; idem Phys. Rev. 69 (1992) 725; idem Phys. Lett. B 299 (1993) 58; S. Kelley, J. L. Lopez, D. V. Nanopoulos, H. Pois and K. Yuan, Phys. Lett. B273 (1991) 423; idem Nucl. Phys. B 398 (1993) 3; W. de Boer, R. Ehret and D. I. Kazakov, IEKP-KA/93-13; D.J. Castano, E.J. Piard, P. Ramond, UFIFT-HEP-93-18.

[6] J. Lopez, D.V. Nanopoulos and H. Pois, Phys. Rev. D47 (1993) 2468; J. Lopez, D.V. Nanopoulos, H. Pois, X. Wang and A. Zichichi, Phys. Lett. B306 (1993) 73; R. Arnowitt and P. Nath, Phys. Rev. Lett. 70 (1993) 3696; R. Arnowitt and P. Nath, Phys. Lett. B 289 (1992) 368; R. G. Roberts and L. Roszkowski, Phys. Lett. B309 (1993) 329; V. Barger, M. Berger and P. Ohmann, MAD/PH/801; G. Kane, C. Kolda, L. Roszkowski and J. Wells, UM-TH-93-24.

[7] P. Fayet, Nucl. Phys. B 90 (1975) 104.

[8] H.P. Nilles, M. Srednicki and D. Wyler, Phys. Lett. B120 (1983) 346; J.M. Frere, D.R.T. Jones and S. Raby, Nucl. Phys. B222 (1983) 11; J.-P. Derendinger and C.A. Savoy, Nucl. Phys. B237 (1984) 307; J. Gunion and H. Haber, Nucl. Phys. B272 (1986) 1; J. Gunion, H. Haber, M. Sher, Nucl. Phys. B306 (1988) 1. 
[9] J. Ellis, J. Gunion, H. Haber, L. Roszkowski and F. Zwirner, Phys. Rev. D39 (1989) 844.

[10] L. Hall, J. Lykken and S. Weinberg, Phys. Rev. D27 (1983) 2359; U. Ellwanger, Phys. Lett. B133 (1983) 187; J.E. Kim and H.P. Nilles, Phys. Lett. B138 (1984) 150; K. Inoue, A. Kakuto and T. Takano, Prog. Theor. Phys. 75 (1986) 664; A. A. Ansel'm and A. A. Johansen, Phys. Lett. B200 (1988) 331; G. Giudice and A. Masiero, Phys. Lett. B206 (1988) 480.

[11] S. Ferrara, D.V. Nanopoulos and C. A. Savoy, Phys. Lett. B123 (1983) 214; J. Polchinski and L. Susskind, Phys. Rev. 26 (1982) 3661; H.P. Nilles, M. Srednicki and D. Wyler, Phys. Lett. B124 (1982) 337; A. B. Lahanas, Phys. Lett. B124 (1982) 341; L. AlvarezGaume, J. Polchinski and M. B. Wise, Nucl. Phys. B221 (1983) 495.

[12] J. Bagger and E. Poppitz, Phys. Rev. Lett. 71 (1993) 2380.

[13] U. Ellwanger and M. Lindner, Phys. Lett. B301 (1993) 365; U. Ellwanger, Phys. Lett. B303 (1993) 271; T. Elliott, S. F. King and P. L. White, Phys. Lett. B305 (1993) 71; T. Elliott, S. F. King and P. L. White, Phys. Lett. B314 (1993) 56; P. N. Pandita, Phys. Lett. B318 (1993) 338.

[14] T. Elliott, S. F. King and P. L. White, Phys. Rev. D49 (1994) 2435.

[15] CDF collaboration: F. Abe et al, Phys. Rev. Lett. 73 (1994) 225; ibid Phys. Rev. D50 (1994) 2966; D0 collaboration: S. Abachi et al, FERMILAB-PUB-354/E (Phys. Rev. Lett. to be published).

[16] U. Ellwanger, M. Rausch de Traubenberg and C. A. Savoy, Phys. Lett. B315 (1993) 331.

[17] Ph. Brax, U. Ellwanger and C. A. Savoy, LPTHE Orsay 94/101, DAMTP/94-98, SaclayT94/. 
[18] U. Ellwanger, M. Rausch de Traubenberg and C. A. Savoy, LPTHE Orsay 95-04, LPT Strasbourg 95-01, SPhT Saclay T95/04. 


\section{Figure Captions}

Figure 1: A contour plot showing allowed values of $\lambda_{0}$ and $k_{0}$ for given values of $h_{t 0}=1,2,3$ (going from left to right) in the $\lambda_{0}-k_{0}$ plane, corresponding to $M_{1 / 2}=500 \mathrm{GeV}$, $\tilde{m}_{0}=2$ with $\left|A_{0} / m_{0}\right|=3$.

Figure 2: Masses of particles as a function of $M_{1 / 2}$. The input parameters are $h_{t 0}=2$, $\left|A_{0} / m_{0}\right|=3, \tilde{m}_{0}=5, \lambda_{0}=0.4, k_{0}=0.275-0.3$. We use $Q=150 \mathrm{GeV}$. Neutralinos (solid lines), charginos (dot-dashed lines), CP-even Higgs (short dashed lines), lighter stop and top quark (both dotted lines), left-handed sleptons (long dashed lines), and gluino (quadruple dashed lines) are displayed. One of the CP-odd Higgs and the charged Higgs bosons (not shown) are roughly degenerate with heavier CP-even Higgs bosons. The remaining CP-odd Higgs boson (not shown) is heavier than the heaviest neutralino. The lightest CP-even Higgs (which has standard model-like couplings), lighter chargino and lightest two neutralinos are all in the LEP2 range for $M_{1 / 2} \lesssim 100 \mathrm{GeV}$.

Figure 3: Masses of particles as a function of $M_{1 / 2}$. The input parameters are $h_{t 0}=0.5$, $\left|A_{0} / m_{0}\right|=4, \tilde{m}_{0}=0.5, \lambda_{0}=0.1, k_{0}=0.07-0.13$. In addition to using $Q=150 \mathrm{GeV}$, as in Fig.2, we also show results for $Q=25 \mathrm{GeV}$, corresponding to minimum values of $M_{1 / 2}=300 \mathrm{GeV}$ and $M_{1 / 2}=125 \mathrm{GeV}$, respectively. Neutralinos (solid lines), charginos (dot-dashed lines), CP-even Higgs (short dashed lines), lighter stop and top quark (both dotted lines), left-handed sleptons (long dashed lines), and gluino (quadruple dashed lines) are displayed. One of the CP-odd Higgs (not shown) is roughly degenerate with the second heaviest CP-even Higgs boson. The remaining CP-odd Higgs boson (not shown) is roughly equal to the lighter stop mass. The lightest CP-even Higgs (which has standard model-like couplings as in Fig. 2 ) has a mass $\lesssim 100 \mathrm{GeV}$. 
This figure "fig1-1.png" is available in "png" format from: http://arxiv.org/ps/hep-ph/9406303v5 
Figure 1

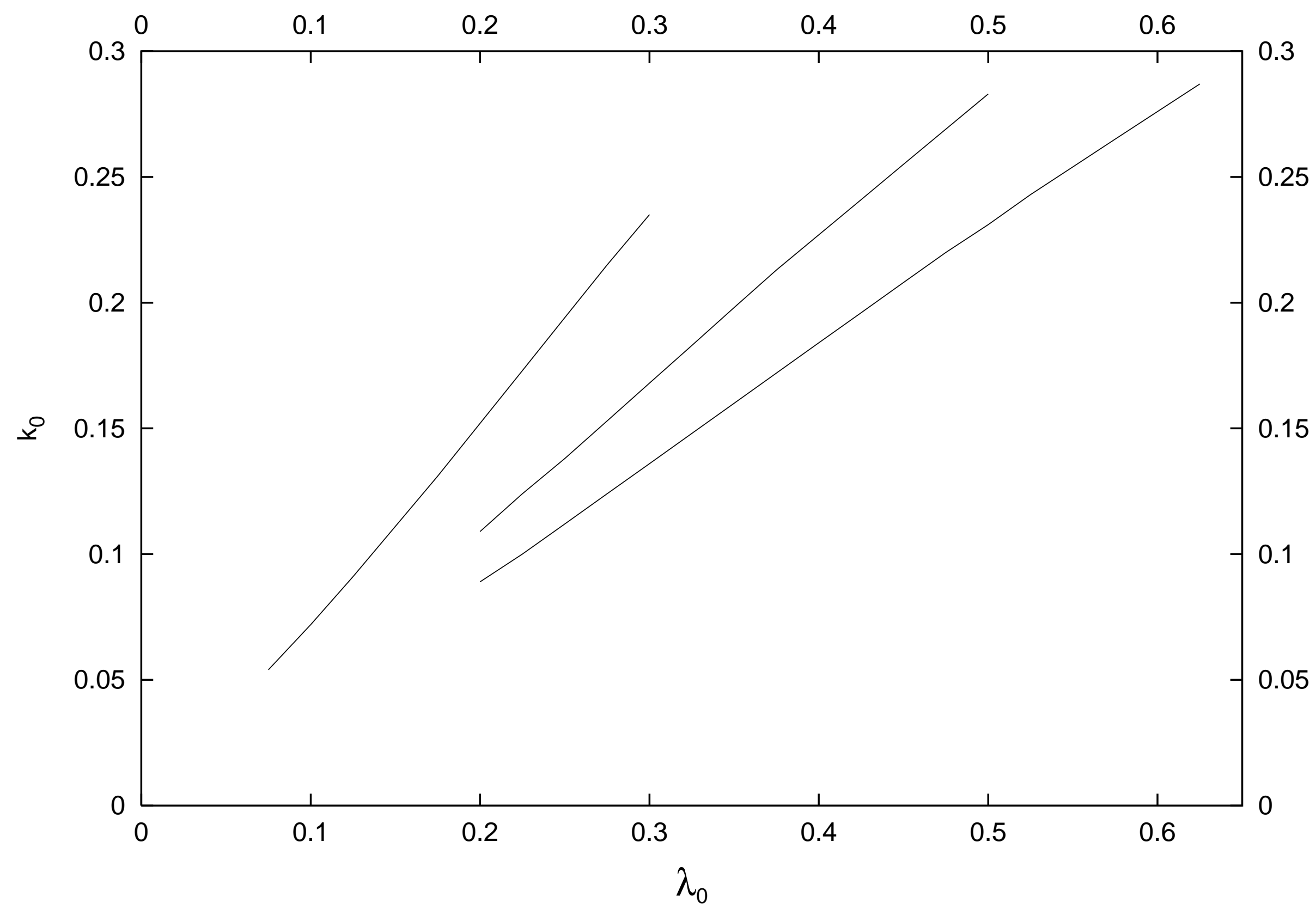


Figure 2

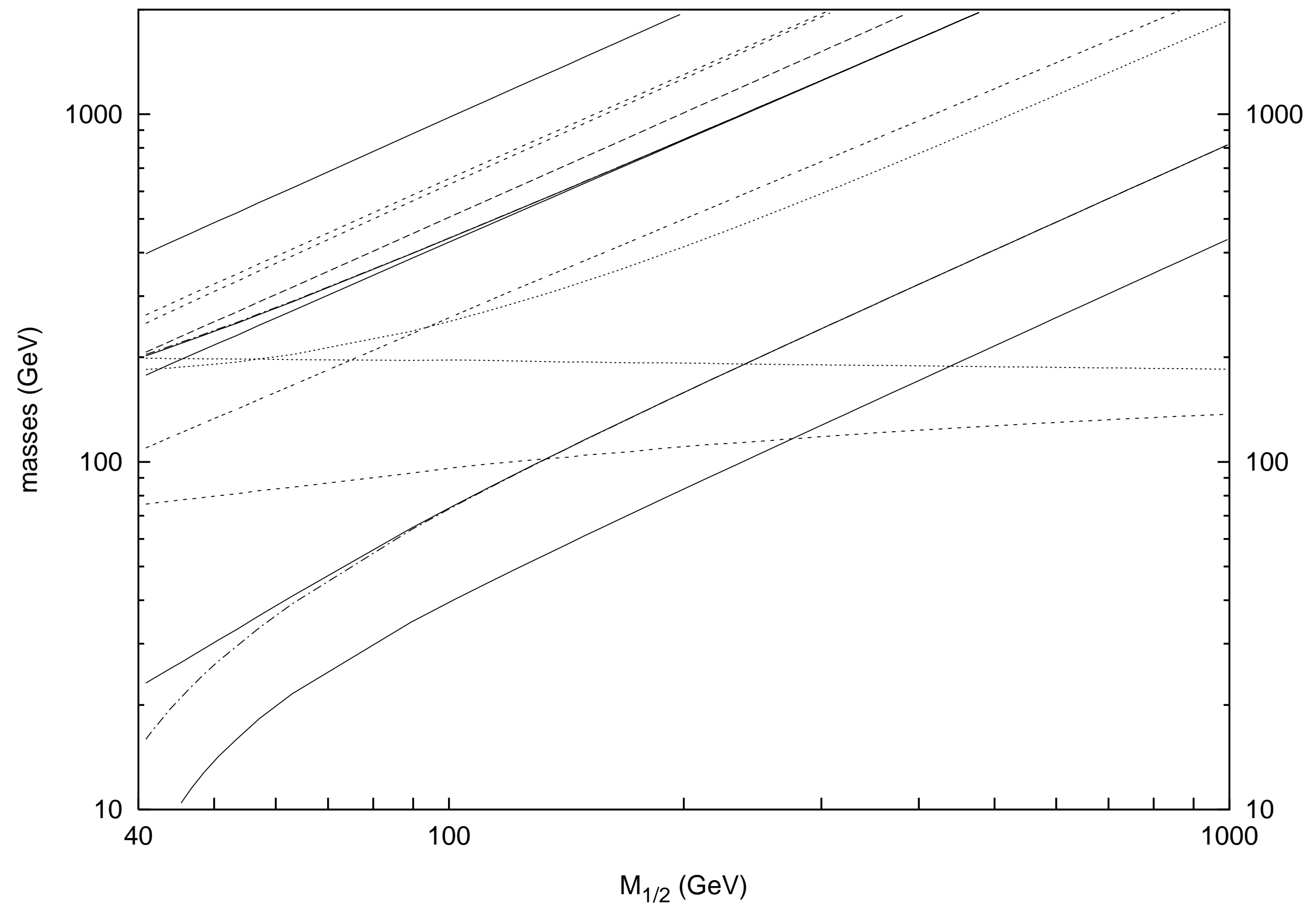


Figure 3

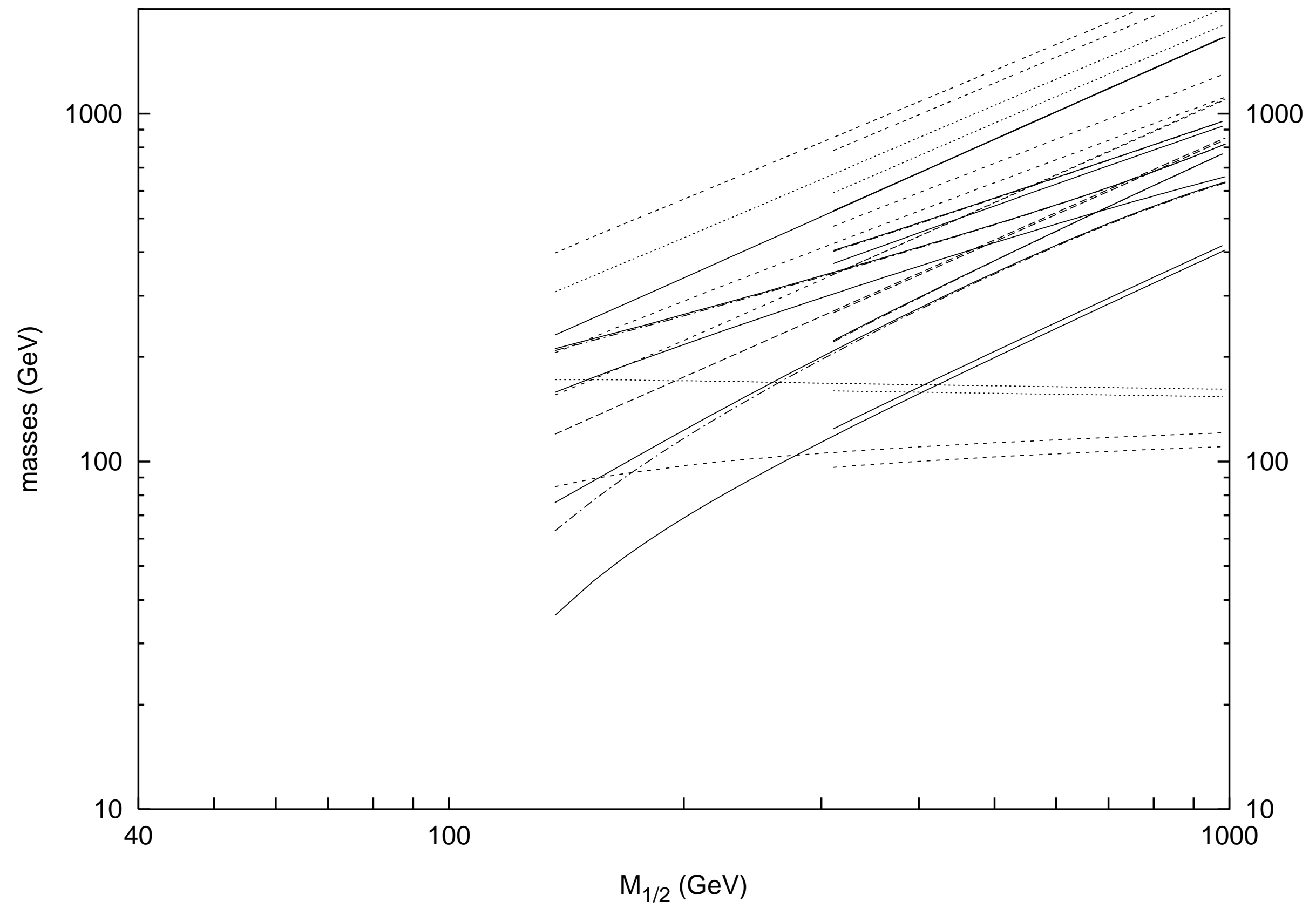

